\title{
LAS CRÍTICAS ANTROPOLÓGICAS A LA TEORÍA INTENCIONAL DEL SIGNIFICADO
}

\author{
Eduardo DE BUSTOS \\ Dpto. de Lógica y filosofía de la ciencia \\ UNED- Madrid
}

\section{Introducción}

Algunas críticas a la teoría intencional del significado proceden de la frustración de las esperanzas iniciales que tal teoría indujo en el campo de las ciencias sociales, especialmente en sociologia y antropología. Al fin y al cabo, la teoría intencional del significado no partía de una concepción formalista del lenguaje, de una imagen del lenguaje como un sistema abstracto de signos, imagen común al estructuralismo y al logicismo semánticos, sino que consideraba las lenguas humanas como depositarias de recursos para la realización de acciones sociales. En la misma concepción del segundo Wittgenstein sobre la comunicación lingüística, en la que hundía sus raices la teoría intencional del significado, se encontraba introducida ya esa dimensión social: el sentido esencial del argumento wittgensteniano contra la posibilidad de un lenguaje privado era que la producción del significado está sujeta necesariamente a la existencia de una realidad social, la formada por el hablante y su auditorio.

Sin embargo, algunos sociólogos y antropólogos se han sentido decepcionados por la orientación mentalista y no social del desarrollo de la teoría intencional del significado. Por ejemplo, la obra de J. Searle ha evolucionado (a la par que la de la filosofía analítica en general) en el sentido de proporcionar una fundamen-

Éndoxa: Series Filosóficas, no 7, 1996, UNED, Madrid:

Eduardo de Bustos: Las criticas antropológicas a la teoría intencional del significado

pp. 5-19. 
tación psicológica (una teoría adecuada de la mente) a la teoría intencional del significado y de la acción, argumentando en favor de un concepto de mente diferente al que sustentan las teorías semánticas corrientes, la teoría computacional de la mente de J. Fodor y otros ${ }^{1}$. En particular, el concepto de mente que J. Searle defiende en sus últimas obras (J. Searle, 1992) es esencialmente intencional (feriomenológico, dicen otros), no reductible y subjetivo.

Una de las críticas más corrientes ${ }^{2}$ es que, en el seno de la teoría intencional del significado, los actos de habla son concebidos como acciones de individuos aislados $o$ independientes, sin tener en cuenta las constricciones sociales a que se encuentran sometidas tales acciones, empezando por la propia constitución social del individuo en cuanto agente: "[la teoría de los actos de habla] fracasa porque construye la acción de forma independiente de su naturaleza reflexiva tanto como consecuencia como causa de formas sociales humanas" (M.Z. Rosaldo, op. cit.). Para el sociólogo, la consecuencia a extraer es que es preciso eliminar, en las investigaciones sociolingüisticas, cualquier referencia a la intencionalidad, considerando la producción de sentido como un proceso social puro, no psicológico.

En general, los argumentos procedentes de las ciencias sociales a la teoría intencional del significado se pueden clasificar en tres grandes apartados:

1 Véase M. Dascal, (1983), Pragmatics and the Philosophy of Mind, 1: Thought in Language, Amsterdam: J. Benjamin.

${ }^{2}$ M. Z. Rosaldo (1982), "The things we do with words», Language in Society, 11, págs. 203-237. 
- Irrelevancia del componente intencional: existen usos lingüísticos en que las intenciones no desempeñan ningún papel, por lo tanto la apelación a ellas para explicar la conducta es vacua.

- Preponderancia de los efectos perlocutivos: en ciertas ocasiones o ámbitos discursivos los efectos perlocutivos de una acción pueden terminar (y explicar) la conducta lingüística.

- Etnocentrismo de la explicación intencional: la apelación explicativa a las intenciones puede ser etnocéntrica, en el sentido de que la noción de intención forma parte de nuestra teoría común para la explicación de la conducta en nuestra cultura, pero puede no ocupar ese lugar central en otras.

\section{El ritualismo lingüístico}

En el lenguaje ritual parece darse un contraejemplo a la teoría intencional del significado. La ausencia de intención, antes que su presencia, parece constituir una condición necesaria para el uso de tal lenguaje. El lenguaje ritual parece tener efecto perlocutivo, a pesar de, o precisamente por, la ausencia de intención de quien lo usa. J. Nuyts ${ }^{3}$ ha analizado el núcleo de esta crítica antropológi$\mathrm{ca}^{4}$, señalando corrrectamente cuáles son sus puntos débiles. Sus observaciones se pueden generalizar del siguiente modo. En todo lenguaje ritual, se da o pretende producir un efecto perlocutivo, especialmente cuando implica una interacción, como en el caso de las prácticas adivinatorias. En este sentido, se puede decir que se

3 J. Nuyts (1994), «The intentional and the socio cultural in language use», Pragmatics and Cognition, 2, 2, págs. 237-268.

4 Formulada, por ejemplo, por J. Dubois, 1987, «Meaning without intention», Papers in Pragmatics, 1,2, págs. 80-122. 
da una de las condiciones de la existencia de comunicación y, por lo tanto, de creación de sentido.

Ahora bien, lo característico del lenguaje ritual es que el efecto perlocutivo no se alcanza, ni se pretende alcanzar, a través del contenido de lo enunciado. Por una parte, a) el significado léxico u oracional puede ser desconocido para el hablante, para su auditorio, o para los dos; por otra, b) aunque parcialmente conocido, puede que no sea utilizado, en cuanto tal contenido, para causar el efecto perlocutivo deseado.

Puede ser que, en el caso más extremo, el significado lingüístico del conjuro, la adivinación, la profecía...sea desconocido tanto para el emisor como para el receptor. Pero, aún así, para que el funcionamiento de la institución sea correcto, es necesario que estén presentes diversos elementos que permiten comprender el ritualismo en términos de la teoría intencional de la acción:

\section{El sentido ritualizado}

- el hablante, u oficiante, debe tener tres clases de intenciones, que convierten su acción en comunicativa, al menos en parte

i. la intención de utilizar a, aunque el contenido linguístico de a puede ser desconocido para él. Esto implica, además, criterios de discriminación con respecto a otras posibles enunciaciones lingüísticas, incluso aunque sean lingüísticamente tan opacas como a para él. En el ritualismo verbal tales criterios de discriminación, precisamente a causa de la opacidad semántica, pueden ser muy estrictos -es una tesis común en historia de la lingüística que el surgimiento de la gramática como sistema codificado de convenciones lingüísticas se debe precisamente a la necesidad de codificar externamente el lenguaje ritualizado.

Los criterios de discriminación entre mensajes están además naturalmente ligados a los criterios de corrección en el uso del lenguaje ritual que, a su vez, son la fuente de legitimación del oficiante, cuando el uso del lenguaje ritual está unido a posiciones sociales de poder o prestigio. 
ii. la intención de utilizar a para causar $\mathbf{b}$, donde $\mathbf{b}$ describe el correspondiente efecto perlocutivo. De la opacidad del lenguaje ritual no se sigue pues la ausencia de objetivos comunicativos por parte del usuario, sino el abandono de que tal conexión esté basada en el reconociminento del significado de $\boldsymbol{b}$, si por tal significado se entiende el significado lingüístico de a.

iii. la intención de que $b$ sea causada, al menos en parte, por la comprensión de a y de ii. Evidentemente, en el caso del ritualismo, el oficiante no puede pretender que $b$ sea causada a través de la comprensión del significado de $a$, entre otras cosas porque a puede ser impenetable para él mismo. Pero sí que es necesario que pretenda que el auditorio reconozca a como muestra diferente de otras enunciaciones rituales posibles y como muestra correcta para la consecución del efecto b correspondiente. En este sentido, se puede afirmar que el oficiante atribuye un contenido, siquiera mínimo, a a, el contenido necesario para que el auditorio capte ii, para que discrimine a y para que tal discriminación le pueda habilitar para reconocer a como correcta o incorrecta.

- Por su parte el auditorio adscribe significado a la acción del oficiante en los siguientes términos

i. considera que el oficiante tiene la intención de causar $\mathbf{b}$, pero tal atribución no puede estar basada en rasgos internos de a, sea su estructura o su significación lingüística, puesto que a puede ser impenetrable para él ( $y$, recuérdese, para el oficiante mismo.)

ii. atribuye al oficiante la intención de causar $b$ a través de la proferencia de a, aunque, una vez más, tal atribución no se basa en la comprensión de a, puesto que la intención del oficiante no puede estar codificada (lexicalizada...) en a.

Como el propio J. Nuyts ha indicado ${ }^{5}$, la tesis de que el lenguaje ritual escapa al ámbito de fenómenos que una teoría intencional del significado puede y debe explicar se basa en una concepción muy simple o esquemática de lo que es la intencionali-

5 J. Nuyts, op. cit. pág. 243. 
dad comunicativa. De acuerdo con esta idea, la intención comunicativa consiste en la intención de causar un efecto perlocutivo $\mathbf{b}$ a través del reconocimiento del contenido significativo de una expresión. Por eso, cuando se destaca el hecho de que, en el lenguaje ritual, tal contenido es inexistente, se pretende haber refutado la propia teoría intencional del significado, y de la acción. Sin embargo, la teoría intencional del significado tiene recursos para integrar tales formas de comunicación a través de una noción más compleja y sutil de intención comunicativa. De hecho, se puede considerar que el ritualismo constituye una forma extrema de comunicación indirecta y manejada con los mismos instrumentos conceptuales que la teoría provee para ese caso.

Una característica común a todas las manifestaciones de comunicación indirecta, ya se trate de actos de habla indirectos, de la ironía o de otras formas de habla figurada, la intención comunicativa del hablante no se manifiesta en el contenido significativo de las proferencias empleadas, sino a través de él. Esto quiere decir que, para reconstruir la intención comunicativa del hablante, el auditorio ha de ignorar la interpretación directa de las palabras del hablante y obtener una interpretación diferente. El proceso que desencadena esta búsqueda tiene que ver con un principio general en la teoría de la interpretación que es el de maximización de las racionalidad comunicativa y, en general, comportamental. Seguramente es un principio de fuertes raíces cognitivas -en última instancia evolutivas, en el sentido de que la atribución de significado ha representado una ventaja adaptativa para la especie humana-, pero que, en lo que atañe a la acción lingüística, establece constricciones sobre el conjunto de posibles interpretaciones. En particular, excluye en principio las interpretaciones que suponen la irracionalidad del sujeto y, en consecuencia, promueve las interpretaciones alternativas, aunque no sean directas, pero que convierten en racional las conducta del sujero, esto es, que hacen esa conducta coherente con la consecución de fines atribuidos por el intérprete de la acción al agente. 
Este principio general de racionalidad interpretativa, y de las máximas que de él se desprenden en el caso de la comunicación lingüística, dan cuenta de los casos en que las intenciones comunicativas de los hablantes no son transparentes, esto es, de los casos en que tales intenciones no están explicitas en la comunciación verbal. En todos esos casos - casi todos-, la inferencia de la intención del hablante requiere un trabajo de interpretación por parte del auditorio. El objetivo es la reconstrucción de una intención comunicativa que haga inteligible la acción lingüistica del hablante, lo que en ese contexto quiere decir que ha de ponerla en conexión con objetivos o fines concretos a través de la expresión de intenciones comunicativas. En ese trabajo adicional de interpretación, el mayor soporte del auditorio no suele estar constituido por el contenido mismo de las acciones del hablante, sino por otros aspectos de la situación comunicativa. Por contenido se entiende el significado convencional de las expresiones utilizadas, en el caso de una acción verbal, que al fin y al cabo es una realidad histórica, social y cultural, Pero esa realidad, en el caso de la interpretación indirecta, no es sino el punto de partida, el trampolín para la consecución de interpretaciones racionalizadoras. El papel fundamental en la determinación de éstas lo juega en muchas ocasiones, además de los principios heurísticos generales, el conocimiento del contexto por parte del intérprete. Es el conocimiento del contexto, en un sentido estrictamente cognitivo - no realista u objetivista-, el que habilita al intérprete para formular hipótesis alternativas sobre el significado de la acción del oyente y dotarla de racionalidad. El resultado es, en muchas ocasiones, que la acción verbal de una hablante difiere radicalmente de lo que sus palabras significan, en el sentido de lo que sus palabras significarían bajo una interpretación directa, también a veces llamada "literal".

Ahora bien, en el caso de los actos de habla indirectos y de otros, se puede decir que existe una realidad relativamente estable que es el significado de la lengua. Es una cuestión discutida si ese significado se encuentra siempre presente, en la conciencia del 
hablante, en el proceso de obtención de la interpretación relevante 0 no ${ }^{6}$. Pero, presente o no a la conciencia del hablante, se trata de una realidad estable, relativamente determinada, de la que puede partir un proceso inferencial que lleve a la interpretación adecuada.

¿Qué sucede en el caso del ritualismo verbal? Lo que ocurre es que el auditorio, e incluso el hablante, no tiene acceso, desde un principio, a esa realidad sistemática que es el significado de la lengua. Luego no puede apoyarse en ella para la producción de un significado indirecto. La adscripción de contenido, por decirlo así, descansa exclusivamente en lo que no es el contenido semántico de la expresión, esto es, por una parte la materialidad fónica del mismo, lo que pueda distinguir a esa producción de otras similares $y$, predominantemente, el contexto institucional del comportamiento verbal ritual. Es ese contexto el que en última instancia fija el contenido de la proferencia ritualizada, sin tener que acudir, como piensa J. Nuyts', a la existencia de una intencion comunicativa previa, la del autor de la fórmula ritual, invocada en forma implícita, en la repetición mecanizada de las fórmulas rituales. Por decirlo así, el auditorio es capaz de conectar directamente el efecto perlocutivo de las expresiones con una intención comunicativa sin pasar por el contenido lingüístico de las expresiones. Pero, por muy esquemática que pueda ser la conducta comunicativa del oficiante, aún encaja en el marco explicativo de la teoría intencional del significado:

C.U.P.)

Véase R. Gibbs, 1994, The Poetics of Mind, Cambridge:

J. Nuyts, op. cit. pág. 244. 
el $\mathrm{H}$ (ablante) trata de producir el efecto perlocutivo b en A(uditorio)

a es un medio adecuado, en la medida del conocimiento de $\mathrm{H}$, para producir $\mathbf{b}$ independientemente del significado de $\mathbf{a}$.

Al proferir a, $\mathrm{H}$ trata de que A produzca o experimente el efecto perlocutivo $b$, mediante el reconocimiento de la intención de $\mathrm{H}$ de producir $\mathbf{b}$

\section{Teoria intencional y efectos perlocutivos}

Otra crítica corriente entre antropólogos y sociólogos a la teoría intencional del significado ${ }^{8}$ es que ésta no coloca el peso de sus explicaciones donde realmente está, esto es, en las consecuencias de la acción verbal. De acuerdo con esta crítica, lo que realmente explicaría el uso lingüístico sería, no la intención comunicativa del hablante, su deseo de expresar tal intención y, mediante ella, causar un determinado efecto perlocutivo, sino el hecho de que efectivamente se producen efectos perlocutivos al usar las expresiones. Dicho de otro modo, la explicación alternativa propuesta tendria el siguiente esquema:

8 A. Duranti, 1988, «Intention, language and social action in a Samoan context», Journal of Pragmatics, 2, págs. 13-33. 
$\mathrm{H}$, al proferir a, causa b
b es, en algún sentido, beneficioso para $\mathrm{H}$, en la medida en que
H es considerado socialmente responsable de las consecuencias de
usar a
b, por tanto, explica la proferencia de a

La argumentación es incorrecta al menos en dos sentidos. En el primero, general, comparte la falacia de muchas explicaciones clasificadas como funcionales: pensar que de una determinada relación causal entre $X$ e $Y$ (entre a $y$ b) se puede concluir que $X$ existe para (causar) Y. El hecho de que una acción tenga resultados beneficiosos para su agente no permite explicar sin más la realizacion de tal acción. Pero, además, existen hechos específicos acerca de la naturaleza de la comunicación lingüística que hacen incorrecta esta interpretación funcionalista: como es bien conocido, la relación entre fuerzas ilocutivas y efectos perlocutivos no es una relación determinista. No sólo porque a una misma fuerza ilocutiva genérica (por ejemplo, la de prometer) le pueden corresponder diferentes efectos perlocutivos (irritar, ilusionar, engañar, convencer, ...), sino porque, y esto lo decisivo, tales efectos perlocutivos no están bajo el control del agente, esto es, porque, a pesar de que el agente puede pretender causar el efecto $b$, el efecto. $b$ puede no producirse.

En esta crítica pues se confunden dos relaciones causales: la que liga la intención del hablante de causar $b$, mediante una acción lingüística, con tal acción lingüística y la relación causal entre la acción y su efecto perlocutivo. Sólo la primera es explicativa de la acción del agente, mientras que la segunda no cae bajo el ámbito de la teoría de la comunicación - pero quizás bajo el ámbito de otra disciplina social. Así, si hay algo que explique la acción de prometer mediante el lenguaje, es la intención del agente 
de conseguir un determinado efecto en el auditorio. Lo que no explica tal acción es que, como resultado, tenga de hecho ese efecto u otro, entre los posibles efectos de la acción en cuestión.

\section{4. ¿Es la teoría intencional del significado una teoría etnocéntri- ca?}

Finalmente, otra crítica de carácter general, dirigida a la teoría intencional del significado, es la que afirma que la importancia asignada a la noción de intención en la explicación de la conducta se debe a la naturaleza de nuestra cultura. Se afirma que la explicación del significado en términos intencionales no es sino una pieza de nuestras teorias del sentido común (folk theories) sobre los fenómenos sociales y mentales. En otras culturas, con otras teorias, es posible que la acción verbal sea conceptualizada y explicada en otros términos. Por ejemplo en términos sociales, de tal modo que las acciones no sean concebidas como la expresión de las intenciones individuales, sino como el producto de la interacción social, que constriñe o determina absolutamente el rango de lo verbalmente realizable en cada momento.

Hay que reconocer que esta crítica tiene un punto de razón en la medida en que las intenciones continúan siendo entidades sospechosas en filosofía de la mente. Sospechosas, sobre todo, de ser incapaces de entrar a formar parte de "auténticas" leyes científicas, lo que, como se sabe, para algunos significa leyes físicas. Pero, más allá de la posible o imposible reducibilidad de las explicaciones intencionales, cabe preguntarse sobre el fundamento de la crítica en cuestión, al menos en sus aspectos metodológicos. Todas nuestras teorías, y no sólo las teorias sobre las acciones humanas, son al fin y al cabo parte y producto de nuestra cultura, lo que no las invalida, al menos en principio, como teorías auténticamente explicativas. En ese sentido, nuestras teorias sobre la acción humana no se encuentran en peor lugar que las teorías sobre los fenómenos naturales. También podría argumentarse que 
nuestra física es etnocéntrica, que explica el movimiento de los objetos en términos de las fuerzas que sobre ellos operan (su masa, su velocidad...) La diferencia más llamativa con respecto a las teorías de la acción es que, en el caso de éstas, tenemos un punto de vista alternativo, el de los propios agentes (en un sentido general, el punto de vista de la primera persona como se suele denominar en filosofía de la mente). Esto plantea problemas metodológicos, e incluso epistemológicos, que no se pueden minimizar. Pero, si la teoría intencional de la acción es la mejor teoría de la que disponemos para dar cuenta de nuestras acciones, en nuestra cultura, no es ilegítimo utilizarla para la explicación de las acciones de los pertenecientes a otras culturas, contrastándolas con sus propias teorias.

Como también ha señalado J. Nuyts ${ }^{9}$, esto no significa la supresión de las variaciones en la conducta comunicativa de las diferentes culturas, ni de la heterogeneidad comunicativa dentro de una propia cultura, para lo que es el caso. En ciertas culturas, o en ciertos ámbitos comunicativos de una cultura, puede darse un mayor peso de los factores sociales en la producción del discurso, en detrimento de la libre expresión de la intencionalidad, que puede ser predominante en otros. Piénsese, por ejemplo, en las fuertes constricciones comunicativas que impone el escenario jurídico en nuestra cultura, y en otras. Nada más lejano a la realidad que considerar los actos de habla de los participantes en un juicio como libre expresión de la intencionalidad individual: el escenario institucional ejerce tales constricciones sobre el uso lingüístico que buena parte de los actos lingüísticos que en él se realizan son asimilables a la conducta o el discurso ritual.

Sin embargo, también en este caso, cabe preguntarse si las intenciones comunicativas no desempeñan un papel explicativo en la conducta de los agentes. Al fin y al cabo, el fiscal, defensor, juez...quieren decir lo que dicen, por mucho que no puedan decir

9 J. Nuyts, op. cit., pág. 251. 
todo lo que quieren. Aún limitadas, sus intenciones comunicativas permiten adscribir significado a sus expresiones, junto con otros factores relevantes. El juez puede declarar cerrado el caso en contra de su voluntad, pero, cuando lo hace, quiere hacer exactamente eso, declarar el caso cerrado.

Estas observaciones matizan lo que hay de cierto y de erróneo en la acusación de ethocentrismo. Lo que hay de erróneo es la tesis, extrema, de que las intenciones no desempeñan un papel explicativo en la conducta de los individuos de otra cultura. Lo que hay de cierto es que la conducta lingüística no siempre es el fruto de la libre expresión de la intencionalidad comunicativa. Efectivamente, en muchos ámbitos discursivos, la formación de esas intenciones comunicativas está limitada, incluso extremadamente limitada, por el marco social e institucional en que se desarrolla la comunicación lingüística, lo que no impide que tales intenciones sigan pudiéndose considerar los legítimos antecedentes causales de la conducta.

\section{Conclusión}

Como sucede en muchas ocasiones, estas críticas a la teoría intencional del significado están motivadas por unas desmesuradas expectativas respecto a su rendimiento teórico. Sin embargo, nada hay más perjudicial para una teoria que ser impulsada más allá de sus ámbitos explicativos, especialmente si se convierte en una teoria sobre toda la complejidad de fenómenos que conforman una realidad social.

Hay que reconocer, sin embargo, que la teoría intencional del significado, al menos en la concepción original de H.P. Grice, alentó esas expectativas: prometía fundamentar las nociones semánticas abstractas sobre la base material de los acontecimientos comunicativos reales, utilizando únicamente conceptos psicológicos básicos, como intención, creencia, deseo... Daba la impresión de que la teoría intencional proporcionaba una explicación adecuada de la producción del sentido en general, de hecho de la creación del 
sentido en la comunicación. De ahí a interpretar que la teoría intencional explicaba todo lo explicable sobre los procesos de constitución del sentido había sólo un paso. Sin embargo, como ya se ha mencionado, diversos críticos pusieron de relieve que la producción de sentido en la comunicación no puede operar sobre el vacío, o sobre la pura intencionalidad del agente. En la comunicación, los participantes se comportan en un marco social de convenciones e instituciones, y de conocimientos, que limitan no sólo la expresión de las intenciones, sino también su propia constitución. En cuanto a lo que se refiere al conocimiento lingüístico, es evidente que no cualquier expresión puede expresar cualquier intención comunicativa $o$, si se quiere decir de otro modo, que la expresión de las intenciones está constreñida por las convenciones (conocimientos) lingüísticas. Constreñida no significa explicada, aunque suponga una determinación parcial. La explicación sólo se produce cuando se comprende que tales contenidos convencionales sólo son activados, en la comunicación real, por las intenciones comunicativas del agente.

Por expresarlo con una analogía física, la pragmática se ocupa de la dinámica del significado, de cómo se actualiza, de cómo cobra vida real el lenguaje en la comunicación. Pero la mecánica del significado, si por tal disciplina se entiende la que se ocupa de la constitución de las convenciones o conocimientos lingüisticos, es objeto de otras disciplinas, sociales, históricas o cognitivas.

En cualquier caso, he presentado una de las posibles formas de determinar lo que es la pragmática y su lugar dentro de los estudios del lenguaje y la comunicación mediante él. Pero más importante que acotar de forma precisa su ámbito explicativo, es señalar lo que la pragmática ha aportado a los estudios sobre el lenguaje, su perspectiva. Existen muchas formas de caracterizar esa perspectiva, casi tantas como definiciones de pragmática, pero, por mi formación, se me permitirá una muy general, que se remonta a la obra de un filósofo que pasa por ser el primer tratado sistemático de filosofía del lenguaje. El filósofo es J. Locke y la obra el Ensayo sobre el entendimiento humano. Allí, Locke introdujo 
por vez primera el término semiótica para designar la disciplina que se había de ocupar de los sistemas de signos (especialmente el lenguaje natural) y de su relación con aquello de lo que eran signos - las ideas, para Locke. El supuesto fundamental que Locke introdujo al diseñar la semiótica es que el lenguaje es un sistema de representación y esa tesis ha dominado la filosofía del lenguaje durante cuatro siglos y la constitución de la lingüística. La tesis semiótica de Locke, la de que el lenguaje codifica información, en cuanto representación de la idea, llevó de forma natural a una creencia universalmente asumida por generaciones de semánticos, la de que el significado es una propiedad intrinseca del signo lingüistico. $Y$ es este el supuesto básico que, en mi opinión, ha venido a modificar o corregir la pragmática contemporánea. 